\title{
The Impact of Misregistration on Change Detection
}

\author{
John R. G. Townshend, Christopher O. Justice, Charlotte Gurney, and James McManus
}

\begin{abstract}
The impact of misregistration of images on the detection of changes in land cover has been evaluated using spatially degraded Landsat MSS images. Attention is focused on simulated images of the normalized difference vegetation index (NDVI) at two of the spatial resolutions of the planned Moderate Resolution Imaging Spectrometer (MODIS), namely 250 and $500 \mathrm{~m}$. In the first of two sets of experiments singledate images from seven diverse areas were first misregistered against themselves and the statistical properties of the differences were analyzed using semivariograms. In general, we would expect that the finer the spatial frequencies present within an image the greater the consequences of misregistration. The results indicate that in the absence of any actual changes to the land surface, the consequences of misregistration were very marked even for subpixel misregistrations. Pairs of images from different time periods were then misregistered. The results showed, that for four of the seven areas, an error equivalent to greater than $50 \%$ of the actual differences in the NDVI as measured by the semivariance, was induced by a misregistration of only one pixel. To achieve an error of only $10 \%$, registration accuracies of 0.2 pixels or less are required. All these four areas were densely covered by vegetation, whereas for the other three more sparsely vegetated areas with semiarid climates, a registration accuracy of between 0.5 and 1.0 pixel were sufficient to achieve an error of $10 \%$ or less. These results indicate that high levels of registration must be achieved by operational monitoring systems if there is to be reliable monitoring of global change.
\end{abstract}

\section{INTRODUCTION}

$\mathbf{T}$ HE usefulness of remotely sensed data is dependent on numerous qualities of sensor systems. Among those which have received particular attention in the past are the selection of spectral bands [3], [27], the radiometric sensitivity of the data [26], and the spatial resolving power of sensors [11], [23]. The usefulness of such data is also dependent on its subsequent processing and increasing attention is being paid to the development of information systems for programs such as the international Earth Observing System (EOS) [5], [1] and the International Geosphere Biosphere Program [12]. With the drive toward more reliable, quantitative estimation of the environment's biophysical properties [6], the absolute and relative calibration of the data has become increasingly important [18]. These characteristics are growing in significance because of current interest in the nature and impact of Global Climate change [10], which demand long-term data sets in order that changes can be reliably observed and documented. Considerable efforts have been devoted

Manuscript received September 5, 1991; revised March 9, 1992. This work was supported in part by NASA Grant NAG-5942. The authors are with the Laboratory for Global Remote Sensing, Department of Geography, University of Maryland, College Park MD 20745.

C. O. Justice is also with the Biospheric Sciences Branch, Goddard Space Flight Center, Greenbelt MD 20715.

IEEE Log Number 9200753. in the last few years to the specification of sensor and data processing systems to provide improved data for these objectives. One major effort relates to the Moderate Resolution Imaging Spectrometer (MODIS-N) [19], which consists of two sensors that are scheduled to be placed on the first U.S. EOS polar platforms. MODIS-N will be the principal EOS sensor for monitoring global changes in the land cover. As part of preparatory work for this sensor, a previous study evaluated the spatial resolution requirements for MODIS-N for monitoring global vegetation [13], [24]. As an outgrowth of this work and through involvement with the MODIS Science Team, it became apparent that insufficient attention was being paid to the geometric characteristics of the data and in particular to the accuracy of image registration.

The registration of data sets to a common spatial framework is an essential precursor to the use of remotely sensed data for monitoring change. If accurate registration between images is not achieved, then spurious differences will be detected, arising merely because different locations are compared instead of differences in properties at the same location between one time and another. Standard texts on remote sensing generally stress the importance of this task and describe various methods for achieving it [4], [15], [16]. But, surprisingly few studies have investigated the consequences of misregistration and we have been able to discover only one quantitative study on this topic [21]. This work showed that classification accuracies achieved using simulated Thematic Mapper data were substantially reduced even if the band-to-band misregistration was only 0.3 of a pixel.

The accuracy of registration is usually quoted in terms of the root mean square (rms) error of the location of ground control points. Values of 0.5 to 1.0 pixels are normally regarded as being satisfactory and visually the results of overlaying two images with this level of misregistration appear acceptable. However the consequences of subpixel misregistration in quantitative terms on multitemporal data sets are unknown.

The immediate stimulus for this research arose from an analysis of images used in previous simulations of MODIS$\mathrm{N}$ [13], [24]. The areas selected included many of the most important global land transformations currently taking place which will impact on the climate or which will be affected by climate change (Table I). In general, we would expect that the finer the spatial frequencies present within an image the greater the consequences of misregistration. Consequently, it is worth noting that the observed changes in land cover occupied relatively small parcels of land (see fig. 6 in [24]) and their total areal extent was usually small. Thus the requirement for good registration is likely to be stringent, whereas those for other vegetational changes such as phenological ones would 
TABLE I

Characteristics of Study Areas and Dates of Images (Further Details of Changes in [24]). Italicized Part of Location Names Indicates Summary Name Used in Graphs and TeXT

\begin{tabular}{|c|c|c|}
\hline Location & Dates of Images & Main Types of Land Transformation \\
\hline Hobbs, New Mexico & $\begin{array}{l}\text { July } 30,1972 \\
\text { August } 27,1984\end{array}$ & $\begin{array}{l}\text { Agricultural encroachment on rangeland. Increasing } \\
\text { pivot irrigation. }\end{array}$ \\
\hline Tucson, Arizona & $\begin{array}{l}\text { May } 30,1976 \\
\text { May } 29,1978\end{array}$ & $\begin{array}{l}\text { Intensification of rangeland uses; changes in ephemeral } \\
\text { stream network. }\end{array}$ \\
\hline Gourma, Mali & $\begin{array}{l}\text { September } 25,1984 \\
\text { September } 28,1985\end{array}$ & $\begin{array}{l}\text { Local substantial increases in green biomass and } \\
\text { increases in drainage density. }\end{array}$ \\
\hline Mato Grosso, Brazil & $\begin{array}{l}\text { July } 28,1972 \\
\text { July } 26,1981\end{array}$ & $\begin{array}{l}\text { Conversion of range forest in large blocks to both } \\
\text { pastoral and arable usage. }\end{array}$ \\
\hline Rondonia, Brazil & $\begin{array}{l}\text { August } 4,1978 \\
\text { August 2, } 1978\end{array}$ & $\begin{array}{l}\text { Conversion of rain forest to agricultural use, in narrow } \\
\text { rectangular grid pattern. }\end{array}$ \\
\hline Richmond, Virginia & $\begin{array}{l}\text { April 29, } 1976 \\
\text { April 28, } 1978\end{array}$ & $\begin{array}{l}\text { Conversion of temperate mixed woodland to agricultural } \\
\text { use. }\end{array}$ \\
\hline $\begin{array}{l}\text { Superior National Forest, } \\
\text { Minnesota }\end{array}$ & $\begin{array}{l}\text { July } 3,1973 \\
\text { June } 18,1984\end{array}$ & Logging fire damage and regrowth of mixed woodland. \\
\hline
\end{tabular}

probably be lower. The areas are not believed to be atypical; in particular, human-induced conversions of land cover tend to be characterized by high spatial frequencies, because of the relatively local scale of human activities. This consideration also suggests that the problems created by misregistration are likely to be greater in the sensing of land surfaces compared with the atmosphere or many ocean properties.

Given these concerns, it was decided to simulate varying degrees of misregistration and to assess their impact on estimates of the true differences between the images. One of the most important properties to be monitored by MODIS-N is the Normalized Difference Vegetation Index (NDVI) which is the spectral ratio of near infrared (NIR) and red spectral bands, namely (NIR-Red)/(NIR+Red). This ratio is sensitive to several vegetation characteristics, including green leaf density, leaf area index, and photosynthetic capacity for some vegetation types [8], [20]. The simulation therefore concentrated on the consequences of progressive misregistration of images in the accuracy of estimates of changes in the NDVI.

\section{DATA SETS AND Methods}

The data sets utilized were the ones used in the aforementioned simulation study on the spatial resolution of MODIS-N for land studies [13] and comprehensive descriptions of their spatial properties can be found elsewhere [24], [25]. The images were coregistered using common ground control points and an rms error of the original MSS data of between 0.5 and 1.0 pixel was achieved using a third-order polynomial equation. This corresponded to values of approximately 40 to $80 \mathrm{~m}$, given the $79 \mathrm{~m}$ pixel size of the MSS data. Since the same ground control points were used in deriving the transformation equation and for estimating the rms error the true value of the latter is likely to be somewhat larger. The data were then degraded using a specially designed filter in an attempt to approximate the performance of the MODIS-
$\mathrm{N}$ sensor [13]. The resultant spatial resolutions were 250 and $500 \mathrm{~m}$, which are the spatial resolutions of the spectral bands, which have been selected for land applications on MODIS$\mathrm{N}$. Consequently the rms errors of registration at these two resolutions were equivalent to 0.16 and 0.32 pixels for the $250 \mathrm{~m}$ data and 0.08 and 0.16 pixels for the $500 \mathrm{~m}$ data sets. Although the data sets were not registered perfectly, the errors of misregistration were apparently relatively small. Errors were estimated using the location of the original ground control points selected for registering the images one to another.

On the basis that the images were well registered, experiments were then performed to assess the consequences of progressive misregistration of the images. The latter was carried out by progressively sliding one image at $45^{\circ}$ to the other and then calculating the resultant changes in the NDVI differences as the images were progressively misregistered. With the images in their original position with zero misregistration, it is assumed that the differences between the NDVI are a result of real differences between the images, although these "real" changes could be a consequence not only of changes in land cover between the two dates but also of other differences such as atmospheric effects. As the images are sequentially misregistered, spurious differences will be introduced and added to the "real" changes in the NDVI.

Before the analysis on the differences between pairs of images from different dates, each of the images was misregistered against itself. The procedure can be envisaged by imagining that each image was copied and the duplicate image was slid across the original in the way described for the pairs from different dates; the resultant differences were then analyzed. The benefit of this procedure is that the images are, by definition, perfectly registered at the start, so that the impacts of any initial misregistration or of other spurious differences such as contrasts in sensor performance or atmospheric interference are eliminated. Consequently, the 
relationship between the spatial structure of the images and the impact of misregistration can be more clearly examined.

In order to estimate the effects of misregistration, the differences between the NDVI images were measured by the semivariance. In the case of misregistering one image against itself, each pixel $X(i, j)$ is compared to a corresponding pixel $X\left(i+d_{i}, j+d_{j}\right)$, where $d_{i}$ is the misregistration from line to line (vertical), and $d_{j}$ is the misregistration from pixel to pixel (horizontal).

The difference between the two values is squared, summed for each comparison, divided by the total number of comparisons and then halved. This gives the semivariance $(S V)$ :

$$
S V=\frac{\sum_{j=1}^{N P} \sum_{i=1}^{N L}\left\{X(i, j)-X\left(i+d_{i}, i+d_{j}\right)\right\}^{2}}{2 N P . N L}
$$

where $N P=$ the number of pixels in a line and $N L=$ the number of lines in an image.

The resultant plots of semivariance against misregistration have properties more akin to the plots of local variance against resolution [29] rather than variogram where the semivariance is plotted against lag. Nevertheless, use of the semivariance has the advantage that the properties of the semivariance plots are well understood from previous work [9]. For example, assuming that we are dealing with a stationary image, then in the experiments involving the sliding of one image over a copy of itself, the semivariance will progressively rise from a value of zero when there is no misregistration $\left(d_{i}=0, d_{j}=0\right)$ and will asymptotically approach the variance $(V)$ (not the semivariance) of the original image.

$$
V=\frac{\sum_{j=1}^{N P} \sum_{i=1}^{N L}\{X(i, j)-\bar{X}\}^{2}}{N P . N L}
$$

where $\bar{X}$ is the mean. At this level of misregistration, the pixels being compared are essentially uncorrelated, so that any further increase in misregistration will have no effect. The number of pixels at which this occurs is called the range.

The results can be normalized by plotting the semivariance values as a proportion of the variance of the image [17]

$$
\text { Normalized } S V=\frac{S V}{V} \text {. }
$$

Values will then tend progressively toward 1.0. If the data are nonstationary (i.e., there is some sort of trend across the image), this assumption will not hold and values could exceed 1.0 or never reach this value. Once results are normalized, differences between the curves due to the different image variances will have been removed and the form of the curve will be solely due to the spatial structure of the image. For images with a substantial amount of high frequency variation the range will be small, whereas if there are large, near uniform areas, the semivariance will rise slowly and should gradually reach the maximum value at a misregistration equivalent to the diameter of the uniform areas.

When we compare images obtained at different times, each pixel in the first image $X(i, j)$ is compared with the corresponding pixel in the second image $Y\left(i+d_{i}, j+d_{j}\right)$. The semivariance $(S V)$ is then:

$$
S V=\frac{\sum_{j=1}^{N P} \sum_{i=1}^{N L}\left\{\left(X(i, j)-Y\left(i+d_{i}, j+d_{j}\right)\right)\right\}^{2}}{2 N P . N L} .
$$

When $d_{i}=0$ and $d_{j}=0$, the images are perfectly registered and the semivariance will then be half the mean square difference between the two images. On the semivariogram this value is represented by the $y$-axis intercept, also known as the "nugget" [9], which represents the actual differences between the images. If there were no differences between the two images then with zero misregistration then the nugget would be zero. Normalization of the semivariance when misregistering two different images is discussed below.

The pairs of images were misregistered by $0.2,0.5,0.8,1.0$, $2,3,5$, and 10 pixels simultaneously in the $x$ and $y$ directions corresponding to corresponding to $71,177,283,354,707$, 1061,1768 , and 3536 meters for the $250-\mathrm{m}$ pixels and 141 , $354,566,707,1414,2121,3536$, and 7071 meters for the $500-\mathrm{m}$ pixels. Emphasis was placed on values close to one pixel, since it is this region that least was understood about the effects of misregistration. Registration of images to map bases or to other images by fractions of pixels is routinely carried out and the rms error, which is achievable for satellite sensing systems, is commonly less than one pixel (e.g., [28], [2]). Achieving such accuracies cannot be conducted by simply sliding images or parts of images across each other by integer amounts. Instead some sort of resampling of one of the images is needed, usually by a procedure like cubic convolution, which provides an estimate of the pixel value at the new pixel locations. Numerical estimates of the consequences of misregistrations for the smallest values may be somewhat inaccurate since the amount of misregistration is comparable to the inherent registration accuracy of the data sets from different dates (see Section II).

\section{Misregistration of Images Against Themselves}

\section{A. Trends of Unnormalized Semivariances}

Fig. 1 shows the results obtained when all 14 images are misregistered against each other using the $500-\mathrm{m}$ pixels. As anticipated, the plots for all images have an overall convex form and the plots with the larger overall semivariances display a steep rise at relatively small increases in misregistration. Substantial differences between the individual pairs of images occur, most notably for the two pairs of images of Superior and Richmond. No simple trends can be observed in relation to obvious vegetation type. All three sparsely vegetated semiarid areas, namely Mali, Hobbs, and Tucson, have relatively low semivariances, but the trends for the tropical rain forest images of Rondonia are similar.

\section{B. Trends of Normalized Semivariances}

If interest lies only in changes in the absolute value of the NDVI, then there is no need to normalize the trends. But 


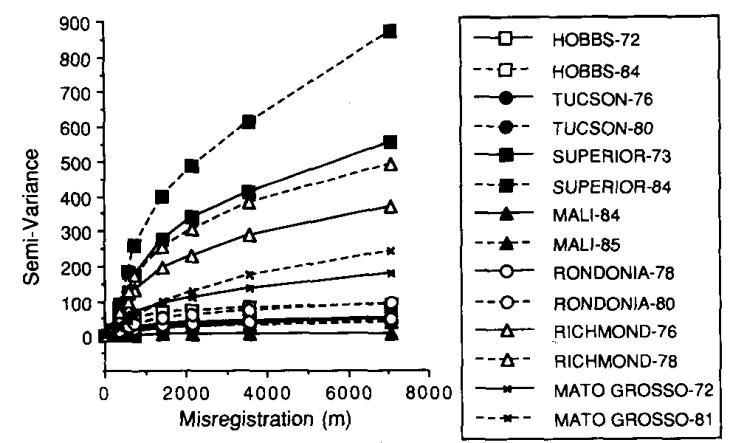

Fig. 1. Increase in semivariance with progressive misregistration, when single date images, with a pixel size of $500 \mathrm{~m}$, are misregistered against themselves.

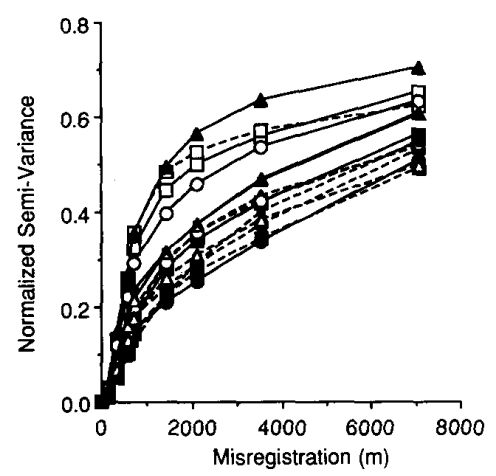

Fig. 2. Increases in normalized semivariance with progressive misregistration, when single date images, with a pixel size of $500 \mathrm{~m}$, are misregistered against themselves (see Fig. 1 for caption).

the absolute changes may be relatively unimportant compared with relative changes. For example, in the second Mali image very considerable variations in vegetation cover are found as a result of localized rainfall [14], but these regionally important variations are only represented by relatively small variations in the absolute value of the NDVI.

The variations in the multidate images were normalized by dividing the semivariances by the variance of the images (Section II), since these represent the maximum values assuming the images are stationary. Hence the maximum values should be 1.0, but as Fig. 2 shows none of the curves have normalized semivariances much above 0.6 . This suggests that much greater misregistration than the maximum considered value of 14 pixels of diagonal movement would be required before the maximum semivariance values could be reached. Alternatively, the maximum theoretical value of $1.0 \mathrm{might}$ not be achievable because the images are nonstationary. Visual examination of the images (Fig. 6 in [24]) supports this supposition for at least three of the areas.

The order of the images on the graph is very different from those obtained without normalization (cf. Fig. 1). For example, the image of Mali for 1984 has moved from the lowest position on the graph to the highest. In those images where the total variances of the images are very small, the effects of misregistration can introduce a proportionately large amount

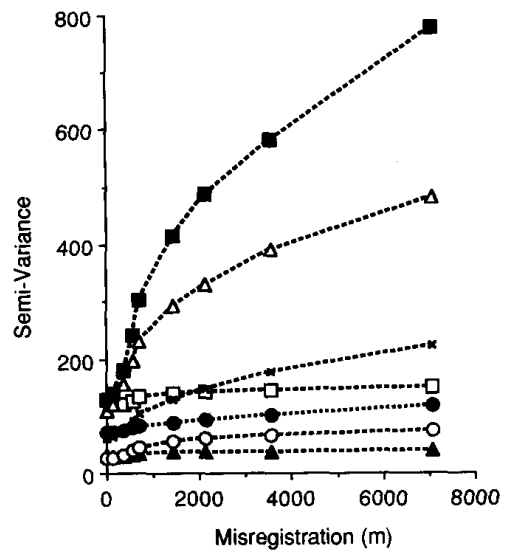

Fig. 3. Increases in semivariance with misregistration, when pairs of NDVI images from two different dates, with a pixel size of $500 \mathrm{~m}$, are misregistered (see Fig. 1 for caption).

of additional variance. The convex form of the plots again indicates the strong effects for small levels of misregistration.

\section{MisRegistration of Change Images}

\section{A. Trends of Unnormalized Semivariances}

Misregistering images from two different times is a more realistic simulation of the influence of registration accuracy on change detection. Fig. 3 shows the resultant curves for the seven different areas. The height at which the curves intercept the $y$-axis, when the misregistration is zero (also known as the nugget) is indicative of the actual changes between the two images. It is notable that the range of nugget values is small, compared with the range of semivariances when misregistration exceeds even $2000 \mathrm{~m}$ (or four pixels). It is notable that the rank order of the curves is very similar to that observed for the images from the individual dates. This is not unexpected, when one considers that for most areas changes between images at the same calendar date are relatively minor. Thus in Fig. 3 the effects of real changes are progressively confused with errors introduced by comparing different locations on the ground, which was exactly the effect observed when misregistering images against themselves.

Careful observation of Fig. 3, indicates that for some areas, there is no immediate increase in the semivariance at the smallest misregistrations, and for the Hobbs area there is a small decline. This presumably arises because the pairs of images were less perfectly registered than indicated by the rms errors of the ground control points (see Section II), and moving them across each other initially leads to a small improvement in registration for subpixel shifts. The failure of the rms error reliably to indicate the accuracy of registration may be a result of using the same ground control points which were used in deriving the transformation equations in the registration process. These do not form an independent sample of locations for estimating registration accuracy and appear to have given a somewhat misleading indication of the true accuracy. 


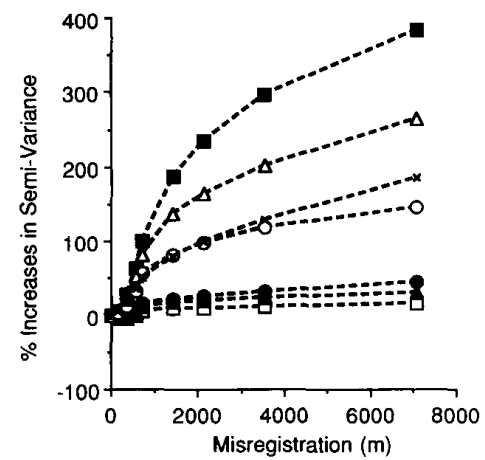

Fig. 4. Percentage increases in semivariance with misregistration, when pairs of NDVI images from two different dates, with a pixel size of $500 \mathrm{~m}$, are misregistered (see Fig. 1 for caption). This and the next three figures indicate the impact of misregistration in detecting spurious changes relative to real changes in the NDVI between the two dates.

\section{B. Proportional Changes Due to Misregistration}

The form of the curves, shown in Fig. 3, indicates the combined effects of real changes in the NDVI, and those due to a lack of spatial registration between the two images. In change detection, it is importnt to ensure that real differences are not being masked by spurious effects, such as those introduced by misregistration. To assess the importance of the error introduced by misregistration compared with actual changes, the percentage increase in semivariance $\left(S V_{m}\right)$ at a misregistration $m$ compared with the semivariance $\left(S V_{0}\right)$ at zero misregistration was calculated as follows:

$$
\% S V_{m}=\frac{\left(S V_{m}-S V_{0}\right)}{S V_{0}} \times 100 \% \text {. }
$$

The resultant plot for the 500-m pixels is shown in Fig. 4 . Even for a few pixels misregistration the percentage increase on semivariance exceeds $100 \%$ for four of the areas.

To better examine the trends, the relationships from Fig. 4 are replotted in Fig. 5 taking the logarithm of the percentage increases in semivariance. Those curves whose values dropped slightly with increasing misregistration, as discussed at the end of the previous subsection have only had values plotted for misregistrations whose semivariances are greater than that at "zero" misregistration, since negative values cannot be plotted on logarithmically scaled axes. Fig. 5 demonstrates dramatically the impact of misregistration. Note that the $50 \%$ line falls at or below the curves of four of the areas for a misregistration of only 1 pixel. In other words, spurious differences greater than $50 \%$ of the true differences between the images have been induced by a misregistration of only one pixel. To achieve an error, less than $10 \%$ of the true differences between the different dates would require a misregistration of only $100 \mathrm{~m}$ or 0.2 of a pixel for these areas. Interestingly, these four areas are all from densely vegetated areas, whereas the other three areas are sparsely vegetated ones with semiarid climates. In Fig. 6 the average values for the densely vegetated and sparsely vegetated areas have been plotted. For the latter areas a misregistration of 1.0 pixel would lead to errors of less than $10 \%$ of the true differences, substantially less than

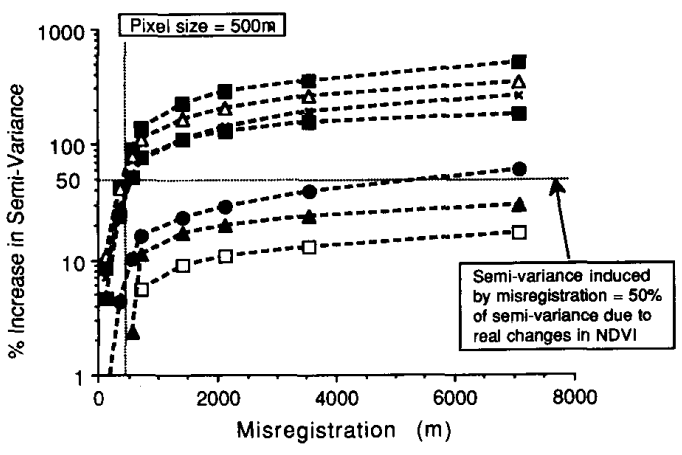

Fig. 5. Semilogarithmic plot of Fig. 4, showing the percentage increases in the semivariance with misregistration, relative to the semivariance with no misregistration, when pairs of NDVI images for two different dates, with a pixel size of $500 \mathrm{~m}$, are misregistered (see Fig. 1 for caption).

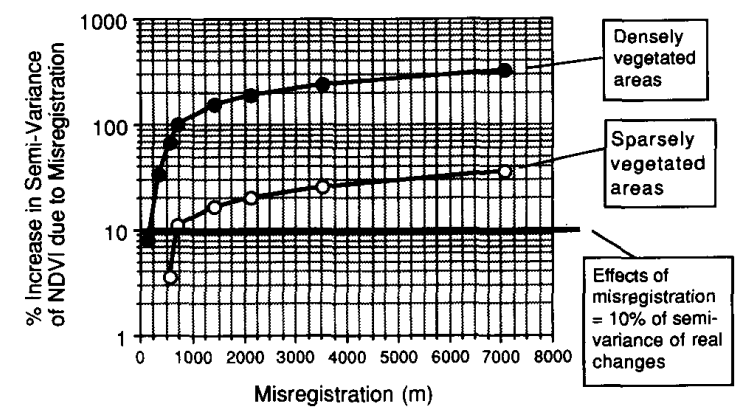

Fig. 6. Average percentage increases in semivariance with misregistration for sparsely and densely vegetated areas relative to the semivariance with no miregistration, when pairs of NDVI images from two different dates, with a pixel size of $500 \mathrm{~m}$, are misregistered.

average value of over $50 \%$ for the more densely vegetated areas.

Percentage changes in semivariance were also derived for a spatial resolution of $250 \mathrm{~m}$. The latter is the resolution of the red and near infrared bands that will be initially sensed by MODIS-N, the other bands for land applications all being sensed at $500 \mathrm{~m} \mathrm{[19].} \mathrm{The} \mathrm{results} \mathrm{indicate} \mathrm{that} \mathrm{slightly}$ coarser misregistrations would be acceptable as measured by the number of pixels (Fig. 7). Nevertheless, on average, a misregistration of only one pixel results in more than a $40 \%$ increase in semivariance for the densely vegetated areas.

\section{CONCLUDING COMMENTS}

Results from simulations indicate the need to achieve high values of registration accuracy, otherwise substantial error terms will be induced when comparisons between images are made for the purposes of detecting land cover changes. Although only images of the NDVI for resolutions of 250 $\mathrm{m}$ and $500 \mathrm{~m}$ have been considered, the evidence from the simulations strongly suggests that misregistration can have a marked effect on the ability of remotely sensed data to detect changes in land cover. Even subpixel misregistrations can have a major impact, and the most marked proportional changes will tend to occur at the finest misregistrations. 


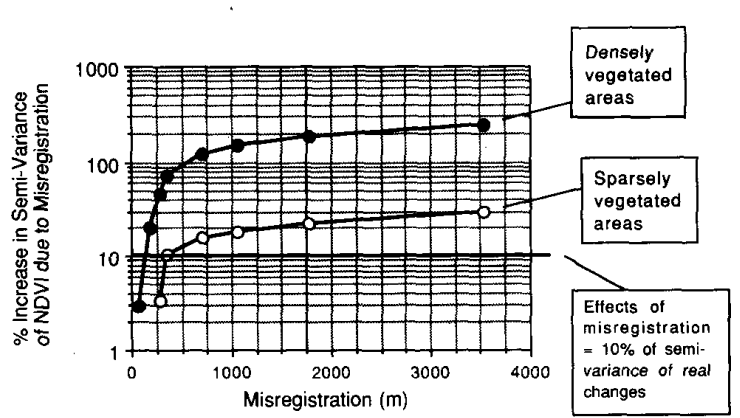

Fig. 7. Average percentage increases in semivariance with misregistration for sparsely and densely vegetated areas relative to the semivariance with no misregistration, when pairs of NDVI images from two different dates, with a pixel size of $250 \mathrm{~m}$, are misregistered.

For four of the areas, a misregistration equivalent to only one pixel induces errors between 50 and $100 \%$ of the true changes in NDVI due to land cover change when the pixel size is $500 \mathrm{~m}$. The potential of the imagery to detect this type of change has therefore been substantially reduced, if not virtually eliminated. To keep errors down to $10 \%$ requires a misregistration accuracy of approx

imately a fifth of a pixel or less for these four areas. For the other three areas a misregistration of between 0.5 and 1.0 pixel would result in errors of $10 \%$ or less. If small geographic areas are being considered, improved accuracy may in part be achieved by additional manual ground control pointing. But for global monitoring instruments such as MODIS-N, interactive manual approaches are impractical for registration. Instead, these results imply the need for highly accurate knowledge of ground location, which can be achieved in part through information on satellite navigation. Such knowledge will need to be much better than that of platforms such as NOAA, SPOT, or Landsat. Alternatively, errors could be reduced by subsequent processing using image-matching methods [22], [7].

One further additional comment should be made concerning the results. We have assumed that all the local errors that are introduced by misregistration are significant. But, if one is making gross regional estimates of change, then it could be argued that spurious positive and negative changes in detected values caused by misregistration will tend to counterbalance each other and the mean value may not be substantially affected. However, the variability around the mean will increase substantially with misregistration. Thus, increases in misregistration will lead to progressively greater overestimates of both positive and negative changes in the NDVI or any other measured spectral property.

Without accurate registration of images the resultant errors are likely to counter markedly the ability of MODIS-N to detect many changes in land cover properties achieved through improvements in instrument properties such as radiometric resolution, spectral bandwidth, and spatial resolution [19]. The results strongly suggest the need to consider the specification of registration accuracy in the design of any remote sensing system, if reliable detection of land cover change is a major objective.

\section{ACKNOWLEDGMENT}

The authors gratefully acknowledge the support of Dr. Vince Salomonson, MODIS Science Team Leader, and helpful critical comments on the analysis by Dr. John Barker of NASA/GSFC and the journal's reviewers.

\section{REFERENCES}

11] A. Ardanuy, D. Han, and V. V. Salomonson, "The Moderate Resolution Imaging Spectrometer (MODIS) Science and data system requirements," IEEE Trans. Geosci. Remote Sensing, vol. 29, pp. 75-88, 1991

[2] A. H. Benny, "Rectification of Landsat-4 Thematic Mapper images without correction data," in 10th International Conference of the Remote Sensing Society. Reading, U.K.: pp. 73-79.

[3] A. S. Benson and S. D. DeGloria, "Interpretation of Landsat-4 Thematic Mapper and Multispectral Scanner data for forest surveys," Photogramm. Engng. and Rem. Sens., vol. 51, pp. 1281-1290, 1985.

[4] R. Bernstein, "Image geometry and rectification," in Manual of Remote Sensing, R. N. Colwell, Ed. Falls Church, VA: American Society of Photogrammetry, 1983, pp. 873-922.

[5] F. C. Billingsley, J. Johnson, E. Greenberg, and M. MacMedan, "Facilitating information transfer in the EOS era," IEEE Trans. Geosci. Remote Sensing, vol. 27, pp. 117-124, 1989.

[6] F. Bretherton, "Earth System Science: A closer view," Report of the Earth Systems Sciences Committee to the NASA Advisory Committee National Aeronautics and Space Administration: Washington, DC, 1988.

[7] A. P. Cracknell and K. Paithoonwattanakij, "Pixel and sub-pixel accuracy in geometrical correction of AVHRR imagery," Int. J. Remote Sensing, vol. 10, no. 4,5, pp. 661-667, 1989

[8] P. J. Curran, "Multispectral remote sensing for estimation of green leaf area index" Phil. Trans. Roy. Soc., Ser. A, vol. 309, pp. 257-270, 1983.

[9] J. C. Davis, Statistics and Data Analysis in Geology. New York: John Wiley and Sons, 1973.

[10] J. A. Eddy, Global Change in the Geosphere-Biosphere, Initial Priorities for an IGBP. Washington, DC: National Academy Press, 1986.

[11] M. R. B. Forshaw, A. Haskell, P. F. Miller, D. J. Stanley, and J. R. G. Townshend, "Spatial resolution of remotely sensed imagery. A review paper," Int. J. Remote Sensing, vol. 4, pp. 497-520, 1983.

[12] IGBP, "The Initial Core Projects, in A Study of Global Change," Rep. no. 12, I. G. B. Programme, Stockholm, Sweden, 1990.

[13] C. O. Justice, B. L. Markham, J. R. G. Townshend, and R. L. Kennard, "Spatial degradation of satellite data," Int. J. Remote Sensing, vol. 10, no. 9, pp. 1539-1561, 1989.

[14] C. O. Justice, J. R. G. Townshend, and D. L. Markham, "MODIS spatial resolution study," Int. J. Remote Sensing, vol. 8, pp. 1119-1121, 1987.

[15] J. R. Mather and G. A. Yoshioka, "The role of climate in distribution of vegetation, Annals of the Association of American Geographers, vol. 58 , no. 1 , pp. $29-41,1968$.

[16] J. G. Moik, Digital Processing of Remotely Sensed Images. Washing ton, DC: NASA, 1980

[17] M. A. Oliver, "Geostatistics and its application to soil science," Soil and Land Use Management, vol. 3, pp. 8-20, 1987.

[18] J. C. Price, "Calibration of satellite radiometers and the comparison of vegetation indices," Remote Sensing Environ., vol. 21, pp. 15-27, 1987.

[19] V. V. Salomonson, W. L. Barnes, P. W. Maymon, H. E. Montgomery, and H. Ostrow, "MODIS: Advanced facility instrument for studies of the Earth as a system," IEEE Trans. Geosci. Remote Sensing, vol. 27, pp. $145-153,1989$.

[20] P. J. Sellers, "Canopy reflectance, photosynthesis, and transpiration," Int J. Remote Sensing, vol. 6, pp. 1335-1371, 1985.

[21] P. H. Swain, V. C. Vanderbilt, and C. D. Jobusch, "A quantitative applications-oriented evaluation of Thematic Mapper Design Specifications," IEEE Trans. Geosci. Remote Sensing, vol. GE-20, pp. 370-377, 1982.

[22] A. K. I. Torlegard, "Some photogrammetric experiments with digital image processing," Photogrammetric Record, vol. 12, pp. 175-196, 1986

[23] J. R. G. Townshend, "The spatial resolving power of earth resources satellites," Progr, in Phys. Geogr., vol. 5, pp. 32-55, 1981.

[24] J. R. G. Townshend and C. O. Justice, "Selecting the spatial resolution of satellite sensors required for global monitoring of land transformations," Int. J. Rem. Sens., vol. 9, pp. 187-236, 1988.

[25] J. R. G. Townshend and C. O. Justice, "The spatial variation of vegetation changes at very coarse scales," Int. J. Rem. Sens., vol. 11, pp. $149-157,1990$. 
[26] C. J. Tucker, "Radiometric resolution for monitoring vegetation. How many bits will do?" NASA Tech. Memo., vol. 80293. Goddard Space Flight Center, Greenbelt, MD: NASA, 1979.

[27] C. J. Tucker, "Remote sensing of leaf water content in the near infrared," Remote Sensing Environment, vol. 10, pp. 23, 1980

[28] R. Welch, T. R. Jordan, and M. Ehlers, "Comparative evaluations of of the geodetic accuracy and cartographic potential of Landsat 4 and Landsat 5 thematic Mapper image data.," Photogramm. Engng. and Rem. Sens., vol. 51, pp. 1249-1267, 1985.

[29] C. E. Woodcock and A. H. Strahler, "The factor of scale in remote sensing," Rem. Sens. Envir., vol. 21, pp. 311-322, 1987.

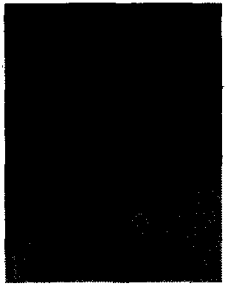

John R. G. Townshend received the Ph.D. degree from the University of London in 1971.

He held posts at the University of Dar es Salaam from 1970 to 1972 and Reading University from 1972 to 1989 , along with visiting positions at Clark University, Massachusetts, from 1977 to 1978 and the NASA Goddard Space Fight Center from 1979 to 1980 . He is currently chairman of the International Geosphere Biosphere Program's Land Cover Working Group.

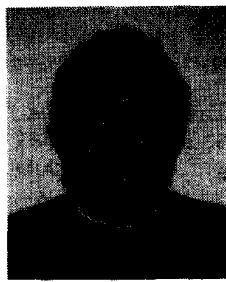

Christopher O. Justice received the Ph.D. degree from Reading University, U.K., in 1977.

He worked at the NASA Goddard Space Fligh Center from 1977 to 1980 . From 1980 to 1982 he worked at the European Space Agency's Earthnet Programme Office at Frascati, Italy. He returned to the University of Maryland, College Park, MD, where he is currently a Research Scholar working on a variety of NASA-funded activities, and he is based in the Biospheric Sciences Branch at the Goddard Space Flight Center. He currently chairs the NASA MODIS Science Team's Land Applications Group.

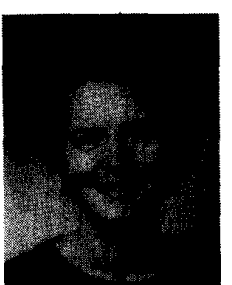

Charlotte Gurney received the B.Sc. degree from the University of Bristol, U.K., in 1976 and the Ph.D. degree from the University of Reading, U.K. in 1980 .

She has held various university research posts and worked for NASA on software development for Landsat data processing. Her main research interest is in the use of contextual information in remotely sensed data. She currently holds a position at the University of Reading.

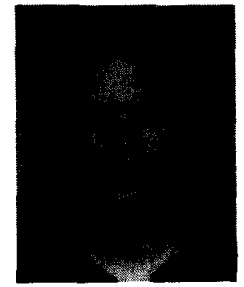

James McManus received the B.S. degree from North Carolina Central University.

$\mathrm{He}$ is currently an M.A. candidate at the University of Maryland, College Park, and works for Hughes STX on the Pilot Land Data System Project. 